\title{
ON A PAPER OF MAURICE SION
}

\author{
MARK MAHOWALD
}

1. Let $M_{0}$ be the set of measures $\mu$ on the real line such that open sets are $\mu^{*}$-measurable. While attempting to find out whether a set $\mu^{*}$-measurable for all $\mu$ in $M_{0}$ is mapped into a similar set by a continuous function of bounded variation, Maurice Sion develops a theory for what he calls variational measure (4). As an application of the theory, he gets conditions on a function $f$ and a set of measures $M$ in order that $f$ map a set, which is $\mu^{*}$-measurable for all $\mu \in M$, into a set of the same kind. In particular he proves for his class $M_{2}$ (def. 2.5), the following theorem (4, § 8.11).

THeorem. If $A$ is measurable for all measures in $M_{2}$ and if $f$ is continuous from the irrationals to $[0,1]$, then $f(A)$ is measurable for all measures in $M_{2}$.

Since all projective sets are continuous images of the irrationals (2, p. 39) and since the existence of a non-measurable projective set is consistent with the axioms of set theory if they are consistent, (1), Sion concludes that Lebesgue measure is not in $M_{2}$.

We prove Sion's result in another way and more importantly, we characterize $M_{2}$ completely with respect to open regular measures. As an application, we prove, without the continuum hypothesis, the existence of a function discontinuous on every set of positive outer measure (Lebesgue).

The author is indebted to William Larkin for many helpful critical comments.

\section{Notation and definitions.}

2.1. A partition, $P(S)$, of a set $S$ is a collection of sets, $E \subset S$, finite in number, pairwise disjoint and whose union is $S$.

2.2. A refinement of a partition, $P_{1}$, is a second partition, $P_{2}$, such that each set in $P_{2}$ is a subset of some set in $P_{1}$.

2.3. An open regular measure is a measure such that each $\mu^{*}$-measurable set has a measurable cover which is a $G_{\delta}$ set.

2.4. $M_{0}=\mid \mu: \mu$ is a measure on $[0,1]$ and open sets are $\mu^{*}$-measurable $\mid$.

2.5. A sequence with property $A$ is a sequence of partitions $P_{n}(S)$ such that
(a) $S \subset[0,1]$ and $0<\mu^{*}(S)<\infty$;
(b) $P_{n+1}$ is a refinement of $P_{n}$;
(c) if $B \subset S$ and $\mu^{*}(B)>0$, then

Received October 17, 1958. 


$$
\lim _{n \rightarrow \infty} \sum_{E \in P_{n}} \mu^{*}(B \cap E)=\infty .
$$

2.6. $M_{2}=\mid \mu: \mu \in M_{0}$ and there does not exist a set $S$ with a partition sequence having property $A \mid$.

2.7. $M_{3}=\mid \mu: \mu \in M_{0}, \mu$ is open regular, there exists a partition sequence $P_{n}([0,1])$ with property $A \mid$.

2.8. A measure will be called non atomic if no single point has positive outer measure.

\section{Conditions implying a measure is not in $\mathbf{M}_{2}$ or is in $\mathbf{M}_{3}$.}

3.1. ThEOREM. If there exists a set $S \subset[0,1]$ of positive outer measure and a bounded function $f$ defined on $S$ discontinuous on every set $E \subset S$ for which $\mu^{*}(E)>0$ and if $\mu$ is open regular, then $\mu \notin M_{2}$.

The proof is long and will be given in $\S 5$.

3.2. Corollary. If $S=[0,1]$ in the theorem, then $\mu \in M_{3}$.

This corollary is an immediate consequence of the proof of the theorem (see 5).

3.3. The following lemma is obtained by a minor modification of the proof of the similar theorem (without the word "bounded") due to Sierpinski and Zygmund (3).

Lemma. There exists a bounded function from the reals to the reals which is discontinuous on every set having the power of the continuum.

3.4. Then we can prove this

THEOREM. If $\mu$ is such that every set of positive outer measure has the power of the continuum and $\mu$ is open regular, then $\mu$ is in $M_{3}$.

Proof. The theorem follows immediately from 3.2 and 3.3.

3.5. Corollary. If there exists one set of positive outer measure such that all subsets of positive outer measure have the power of the continuum and if $\mu$ is open regular for all subsets of this set, then $\mu \notin M_{2}$.

3.6. Corollary. Under the continuum hypothesis: If $\mu$ is non-atomic and open regular, then $\mu$ is in $M_{3}$. If there exists a subset of positive outer measure such that every single point subset has measure zero, then $\mu \notin M_{2}$.

Proof. If a measure is non-atomic then every countable set has measure zero. The continuum hypothesis then implies every set having positive outer measure has the power of the continuum and 3.4 and 3.5 prove the theorem.

3.7. Every measure on the subsets of the unit interval is either in $M_{3}$ or it is not. The definition of $M_{3}$, which enables one to decide whether or not a measure is in $M_{3}$, does not depend on the continuum hypothesis, that is, the 
definition makes sense if the hypothesis is true or false. Now, if there exists a non-atomic, open regular measure not in $M_{3}$, then this can be shown by a set theoretic argument. Such an argument with corollary 3.6 would be a proof from set theory of the proposition: the continuum hypothesis is false. Gödel (1) has shown that this cannot be proven with such an argument. Therefore, all open regular, non-atomic measures are in $M_{3}$, that is, we can improve 3.6 to the following

THEOREM. If $\mu$ is open regular and non-atomic, then $\mu \in M_{3}$.

3.8. We can restate this by this

THEOREM. There are no $\sigma$-finite open regular measures in $M_{2}$. Lebesgue measure is not in $M_{2}$ but it is in $M_{3}$.

\section{A converse to Theorem 3.1.}

4.1. Theorem. If $\mu$ is an open regular measure not in $M_{2}$ and $S$ is a set with a partition sequence having property $A$, then there is a function defined on $S$ which is discontinuous on every subset $E$ of $S$ such that $\mu^{*}(E)>0$.

Proof. For $\mu$, there exists a sequence $P_{m}(S)$ of partitions with property $A$. Let $F_{11}, \ldots, F_{k 1}$ be a numbering of the sets of $P_{1}$. Let $n_{1}$ be the smallest integer larger than $\log _{2} k$. Define

$$
f_{1}(x)=(i-1) / 2^{n_{1}}
$$

for $x \in F_{i 1}$, for $i=1, \ldots, k$.

Suppose for $m-1$ we have defined $n_{m-1}$, a numbering, $F_{i, m-1}$, for the partition $P_{m-1}$, and $f_{m-1}$. The induction step will be defined as follows:

Let $p_{m}=\max _{q} j_{q}$, where $j_{q}$ is the number of sets in $P_{m}$ which are subsets of $F_{q, m-1}$. Let $h_{m}$ be the smallest integer greater than $\log _{2}\left(p_{m}+2\right)$. Let $n_{m}=$ $h_{m}+n_{m-1}$. Let $F_{i m}$, for

$$
i=(q-1) 2^{h_{m}}+1, \ldots,(q-1) 2^{h_{m}}+j_{q}
$$

and

$$
q=1, \ldots, 2^{n_{m-1}},
$$

be a numbering of the sets of $P_{m}$ which are subsets of $F_{q, m-1}$. If $F_{i m}$ does not appear in this numbering, then $F_{i m}=\phi$. Then define

$$
f_{m}(x)=(i-1) / 2^{n_{m}} \quad \text { for } \quad x \in F_{i m} .
$$

The sequence $f_{m}$ is monotonically non-decreasing and is uniformly bounded by one. Therefore there exists a limit function $f_{0}$.

For $m$ fixed, our choice of $h_{m}$ assures us that

$$
\left|x: f_{m}(x)=\left(q 2^{h_{m}}-1\right) / 2^{n_{m}}\right|=\phi
$$

since it would equal

$$
F_{q 2^{h_{m}, m}}
$$


which is empty. As a consequence, we have: if

$$
f_{0}(x)<\left(q 2^{h_{m}}\right) / 2^{n_{m}}
$$

for any $m$ and

$$
q=1, \ldots, 2^{n_{m-1}}
$$

then

$$
f_{0}(x) \leqslant\left(q 2^{h_{m}}-1\right) / 2^{n_{m}}
$$

Therefore

$$
F_{\text {im }}=\left|x:\left[i-1-\left(1 / 2^{n_{m+1}}\right)\right] / 2^{n_{m}}<f_{0}(x)<i / 2^{n_{m}}\right| .
$$

Now suppose there exists a set $B \subset S$ such that $\mu^{*}(B)>0$ and such that $f_{0}$ is continuous on $B$. Since $F_{i m}$ is the inverse image of an open set, $F_{i m} \cap B$ is open in $B$, that is, there exists an open set $U_{i m}$ such that $F_{i m} \cap B=U_{i m} \cap B$. Let $U_{i j m}=U_{i m} \cap U_{j m}$ for $i \neq j$ and $U_{j j m}=\phi$ and let $V_{i m}=U_{i m}-U_{j} U_{i j m}$. Clearly $V$ is pairwise disjoint for $m$ fixed. Also, since $F$ is pairwise disjoint for $m$ fixed, no point of $F_{j m} \cap B$ can be in $U_{i m}$ for $i \neq j$. Therefore, we have $V_{i m} \cap B=F_{i m} \cap B$. Hence we can choose a measurable cover of $F_{i m} \cap B, C_{i m}$, which is a subset of $V_{i m}$. Therefore, $C$ is pairwise disjoint and

$$
\sum_{i} \mu^{*}\left(F_{i m} \cap B\right)=\sum_{i} \mu\left(C_{i m}\right)=\mu\left(U_{i} C_{i m}\right)=\mu^{*}(B) .
$$

Since $m$ is arbitrary, we have

$$
\lim _{m \rightarrow \infty} \sum_{i} \mu^{*}\left(F_{i m} \cap B\right)=\mu^{*}(B)
$$

but this is just

$$
\lim _{m \rightarrow \infty} \sum_{E \in P m} \mu^{*}(B \cap E)=\mu^{*}(B) \leqslant \mu^{*}(S)<\infty .
$$

This contradiction proves the theorem:

4.2. Corollary. For every $\mu \in M_{3}$, there exists a function discontinuous on every set having positive outer measure. In particular there exists such a function for Lebesgue measure.

5. Proof of Theorem 3.1. Let $f$ be the function described in the theorem. Since $f$ is bounded we can suppose that $0 \leqslant f(x) \leqslant 1$. Let $E_{n i}=\mid x: i / 2^{n} \leqslant$ $f(x)<(i+1) / 2^{n} \mid, i=0, \ldots, 2^{n}$. Set $P_{n}(S)=\left\{E_{n i}\right\}$; we shall prove that this sequence has the property $A$. The facts that $P$ is a partition and that $P_{n+1}$ is a refinement of $P_{n}$ are clear. We need only show that, for any $B \subset S$ for which $\mu^{*}(B)>0$, we have

$$
\lim _{n \rightarrow \infty} \sum_{i=0}^{2^{n}} \mu^{*}\left(E_{n i} \cap E\right)=\infty .
$$

Assume that there exists a set $E \subset S$ such that

$$
0<\lim _{n \rightarrow \infty} \sum_{i} \mu^{*}\left(E_{n i} \cap E\right)=a<\infty .
$$


We then shall prove that there exists a subset of $E$ having positive outer measure and on which $f$ is continuous and this contradiction will prove the theorem.

Subadditivity of $\mu^{*}$ implies that the limit in (1) approaches $a$ from below. Therefore, there exists an $N$ such that $n \geqslant N$ implies, for $a / 10>\epsilon>0$,

$$
a-\epsilon \leqslant \sum_{i} \mu^{*}\left(E_{n i} \cap E\right) \leqslant a
$$

and in particular

$$
a-\epsilon \leqslant \sum_{j} \mu^{*}\left(E_{N j} \cap E\right) \leqslant a .
$$

Let $B_{N j}$ be a measurable cover of $E \cap E_{N j}$. If $E_{n i}$ is a subset of $E_{N \jmath}$, we shall write $E_{n i}$, and we shall designate a measurable cover of $E \cap E_{n i j}$ by $B_{n i j}$. It is easily shown that the sets $B_{n i j}, n \geqslant N$, can be so determined that if $I$ is a set of integers such that $\cup_{i \epsilon I} E_{m i}=E_{n k}$, then $\cup_{i \epsilon I} B_{m i j}=B_{n k j}$.

We next derive measurable sets $H_{n i j}$ contained in $B_{n i j}$, disjoint for each fixed pair $n, j$ and with

$$
a-2 \epsilon \leqslant \sum_{j, i} H_{n i j} \leqslant a
$$

Let

$$
\begin{aligned}
B_{n i k j} & =B_{n i j} \cap B_{n k j} & & i \neq k \\
& =\phi & & i=k
\end{aligned}
$$

and let $H_{n i j}=B_{n i j}-\cup_{k} B_{n i k j}$. Since, for $n>N$,

$$
a-\epsilon \leqslant \sum_{j} \mu\left(B_{N j}\right)=\sum_{j} \mu\left(\cup_{i} B_{n i j}\right) \leqslant a
$$

and

$$
a-\epsilon \leqslant \sum_{j, i} \mu\left(B_{n i j}\right) \leqslant a
$$

we have

$$
\sum_{j}\left[\sum_{i} \mu\left(B_{n i j}\right)-\mu\left(\bigcup_{i} B_{n i j}\right)\right]=\sum_{j, i} \mu\left(\cup_{k} B_{n i k j}\right) \leqslant \epsilon .
$$

From this and the definition of $H$, we have

$$
a-2 \epsilon \leqslant \sum_{j, i} \mu\left(H_{n i j}\right) \leqslant a
$$

for all $n>N$. By the choice of $B, \cup_{i} H_{n i \rho}$ is monotonically decreasing as a function of $n$ for each $j$. Letting $H_{j}=\cap_{n} \cup_{i} H_{n i j}$, we have from (3)

$$
\sum_{j} \mu\left(H_{j}\right) \geqslant a-2 \epsilon \text {. }
$$

We next obtain formulas analagous to (3) and (4) with the sets $H_{n i j}$ replaced by open sets. Let $V_{n i j}$ be an open cover of $H_{n i j}$ such that

$$
\mu\left(V_{n i j}\right) \subset \mu\left(H_{n i j}\right)+\epsilon / 2^{i}\left(2^{N}+1\right)
$$


and if $I$ is a set of integers such that $\cup_{i \in I} E_{m i}=E_{n k}$, then $\bigcup_{i \in I} V_{m i}, \subset V_{n k}$. Such a cover exists because of the open regularity of $\mu$. Then for every $n>N$ we have

$$
\begin{aligned}
& a-2 \epsilon \leqslant \sum_{i, j} \mu\left(H_{n i j}\right)=\sum_{j} \mu\left(\cup_{i} H_{n i j}\right) \leqslant \sum_{j} \mu\left(\bigcup_{i} V_{n i j}\right) \\
& \leqslant \sum_{i, j} \mu\left(V_{n i j}\right) \leqslant \sum_{i, j} \mu\left(H_{n i j}\right)+\epsilon \leqslant a+\epsilon .
\end{aligned}
$$

Therefore

$$
\sum_{i, j} \mu\left(V_{n i j}\right)-\sum_{j} \mu\left(\cup_{i} V_{n i j}\right) \leqslant 3 \epsilon .
$$

Using notation analogous to that of (2), letting $U_{n i j}=V_{n i j}-\cup_{k} V_{n i k}$, and using the same argument which leads to (3) and (4), we have

$$
\sum_{j, i} \mu\left(\cup_{k} V_{n i k j}\right) \leqslant 3 \epsilon \text { and } a-5 \epsilon \leqslant \sum_{j, i} \mu\left(U_{n i j}\right) \leqslant a+\epsilon
$$

for all $n \geqslant N$.

By the choice of $V, \cup_{i} U_{n i j}$ is monotonically decreasing as a function of $n$ for each $j$. Letting $U_{j}=\cap_{n} \cup_{i} U_{n i j}$, we have from (5)

$$
\lim _{n \rightarrow \infty} \sum_{j, i} \mu\left(U_{n i j}\right)=\sum_{j} \lim _{n \rightarrow \infty} \mu\left(\bigcup_{i} U_{n i j}\right)=\sum_{\varepsilon} \mu\left(U_{j}\right) \geqslant a-5 \epsilon .
$$

We shall now show

$$
\sum_{j} \mu\left(U_{j} \cap H_{j}\right) \geqslant a-8 \epsilon
$$

Since

and

$$
\mu\left(V_{N j}\right)=\mu\left(V_{N j}-\left(U_{j} \cup H_{j}\right)\right)+\mu\left(\mathrm{H}_{j} \cup U_{j}\right)
$$

we have

$$
\mu\left(H_{j} \cup U_{j}\right)=\mu\left(\mathrm{H}_{j}\right)+\mu\left(U_{j}\right)-\mu\left(H_{j} \cap U_{j}\right),
$$

$$
\sum_{j}\left[\mu\left(H_{j}\right)+\mu\left(U_{j}\right)-\mu\left(H_{j} \cap U_{j}\right)\right] \leqslant \sum \mu\left(V_{N j}\right) \leqslant a+\epsilon
$$

or

$$
a-2 \epsilon+a-5 \epsilon-\sum_{j} \mu\left(H_{j} \cap U_{j}\right) \leqslant a+\epsilon .
$$

This yields (7).

Pick a $j$ such that $\mu\left(U_{j} \cap H_{j}\right)>0$. Then

$$
\mu^{*}\left(\mathrm{E} \cap E_{N j} \cap U_{j}\right) \geqslant \mu\left(U_{j} \cap H_{j}\right)>0 .
$$

Let $C=E \cap E_{N j} \cap U_{j}$. Then for arbitrary but fixed $n>N$ we have

$$
V_{n i j} \cap V_{n k j} \cap C=\phi \text { for } i=k \text {. }
$$

We shall show that $f$ is continuous on $C$. Let $\delta>0$ be given. Then there exists an $n$ such that $2^{-n}<\delta$. Let $x_{0}$ be in $C$. Then

$$
\left|f\left(x_{0}\right)-f(x)\right|<2^{-n}<\delta
$$


for all $x$ in $V_{n i j} \cap C$ where $i$ is such that $x_{0}$ is in $E_{n i}$. Therefore $f$ is continuous on $C$ contrary to hypothesis on $f$. This contradiction proves the theorem.

\section{REFERENCES}

1. K. Gödel, The consistency of the axiom of choice and of the generalized continuum-hypothesis, Proc. Nat. Acad. Sci. U.S.A., 24 (1938), 556-7.

2. W. Sierpinski, Les ensembles projectifs et analytiques, Mémorial des Sciences Mathématiques, no. $112(1950)$.

3. W. Sierpinski and A. Zygmund, Sur une fonction qui est discontinue sur tout ensemble de puissance du continu, Fund. Math., 4 (1923), 316-18.

4. Maurice Sion, Variational Measure, Trans. Amer. Math. Soc., 83 (1956), 205-21.

Xavier University 